\title{
Intranuclear cascade models lack dynamic flow
}

\author{
Joseph J. Molitoris* and Horst Stöcker* \\ National Superconducting Cyclotron Laboratory and Department of Physics and Astronomy, \\ Michigan State University, East Lansing, Michigan 48824 \\ and Institut für Theoretische Physik, Johann Wolfgang Goethe Universität D-6000 Frankfurt am Main, Federal Republic of Germany \\ Hans- ̊̊ke Gustafsson ${ }^{\dagger}$ \\ Gesellschaft für Schwerionenforschung, D-6100 Darmstadt 11, Federal Republic of Germany \\ and Department of Physics, University of Lund, S-22362 Lund, Sweden \\ Joseph Cugnon ${ }^{\ddagger}$ and Denis L'Hôte \\ Département de Physique Nucléaire/Moyennes Energies, Centre d'Etudes Nucléaires-Saclay, \\ F-91191 Gif-sur-Yvette Cedex, France \\ (Received 28 May 1985)
}

\begin{abstract}
We study the recent claim that the intranuclear cascade model exhibits collective sidewards flow. 4000 intranuclear cascade simulations of the reaction $\mathrm{Nb}(400 \mathrm{MeV} /$ nucleon $)+\mathrm{Nb}$ are performed employing bound and unbound versions of the Cugnon cascade. We show that instability of the target and projectile nuclei in the unbound cascade produces substantial spurious sidewards flow angles, for spectators as well as for participants. Once the nuclear binding is included, the peak of the flow angle distributions for the participants alone is reduced from $35^{\circ}$ to $17^{\circ}$. The theoretical "data" are subjected to the experimental multiplicity and efficiency cuts of the plastic ball $4 \pi$ electronic spectrometer system. The flow angular distributions obtained from the bound cascade-with spectators and participants subjected to the plastic ball filter-are forward peaked, in contrast to the plastic ball data. We discuss the uncertainties encountered with the application of the experimental efficiency and multiplicity filter. The influence of the Pauli principle on the flow is also discussed. The lack of flow effects in the cascade model clearly reflects the absence of the nuclear compression energy that can cause substantially larger collective sidewards motion-there is too little intrinsic pressure built up in the cascade model.
\end{abstract}

\section{INTRODUCTION}

Cugnon and L'Hôte have recently claimed ${ }^{1}$ that the intranuclear cascade model developed in Liege ${ }^{2}$-from here on referred to as the Cugnon cascade for brevity-can exhibit collective sidewards flow, in qualitative agreement with the $4 \pi$ exclusive data on $\mathrm{Nb}(400$ $\mathrm{MeV} /$ nucleon) $+\mathrm{Nb}$ obtained by the GSI/LBL plastic ball group. ${ }^{3}$ Collective sidewards flow had originally been predicted by macroscopic nuclear fluid dynamics ${ }^{4}$ and has since then received much theoretical attention because of its possible connection to the nuclear matter equation of state. Calculations done using the intranuclear cascade code of Yariv and Fraenkel ${ }^{5}$ did not exhibit the large sidewards flow angles observed experimentally. ${ }^{3}$ Even more surprising, calculations done with the Cugnon intranuclear cascade code by other authors ${ }^{4,6}$ also produced little flow. On the other hand, different microscopic models such as the classical equations of motion, ${ }^{7}$ the VlasovUehling-Uhlenbeck (VUU) theory, ${ }^{6,8}$ and the time dependent Dirac approach, ${ }^{9}$ which all explicitly incorporate a compressional energy, do predict sidewards flow. In fact, the VUU approach has recently been employed to extract the nuclear equation of state from the experimentally observed flow angular distributions and transverse momentum transfers. ${ }^{6,8}$ Here we underline the fact that-in spite of uncertainties due to a simplified experimental filter- the intranuclear cascade model does exhibit too little dynamical flow and explain why Cugnon and L'Hôte ${ }^{1}$ originally overestimated the flow.

\section{THE INTRANUCLEAR CASCADE MODEL}

Historically, the intranuclear cascade idea is due to Serber. ${ }^{10}$ His idea was that nuclear reactions at high energies might be understood in terms of a simple picture different from the description needed at low energies: $\mathrm{Be}$ cause the collision time between an incident high energy nucleon and a nucleon in the nucleus is short compared to the time between collisions of the nucleons in the nucleus, he inferred that the high energy reaction could be modeled as a cascade process. Collisions occur between the incident particle and those particles which are directly struck in the nucleus. This model was first investigated in two dimensions in 1948 by Goldberger ${ }^{11}$ who performed his calculations by hand for the case of high energy neutrons interacting with heavy nuclei. The first fully threedimensional calculations were done by Metropolis et al. ${ }^{12}$ in 1958 for incident protons and neutrons using the MANIAC computer; they also added a second stage to the cascade calculation during which the excited residual nucleus evaporates particles, as had also been suggested by Serber. ${ }^{10}$ 
Many others have contributed to the development of the intranuclear cascade model. ${ }^{12,13}$ The two most widely used cascade codes in the field of high energy heavy ion reactions are due to Yariv and Fraenkel ${ }^{5}$ and Cugnon, Vandermeulen, Mizutani, and Kińet. ${ }^{2}$ What is the intranuclear cascade model as it is used in these codes? It is a microscopic simulation of a nuclear reaction at high bombarding energies. Nuclear collisions are treated as a superposition of independent two-body nucleon-nucleon collisions. Nucleons move on straight line trajectories (since there is no field) until they collide with a probability given by the free nucleon-nucleon scattering cross section. The creation of deltas, pions, and other particles and the interaction of all these particles occurs according to experimental cross sections. These intranuclear cascade models ${ }^{2,5}$ incorporate relativistic kinematics. Target and projectile nucleons are initialized in configuration and momentum space with random Fermi momenta and then Lorentz boosted to an appropriate frame, where the collision simulation proceeds. Momentum and energy are conserved in the particle-particle interactions and the evolution of the system is followed up to a time where the majority of interactions have ceased. Thus the intranuclear cascade model may loosely be viewed as a solution to the Boltzmann equation without mean-field or sophisticated Pauli blocking factors. Collisions are only Pauli blocked according to a simple criterion, say if the total center of mass energy is less than the Fermi energy in ground state nuclear matter ${ }^{2}$ or if the outgoing particle would scatter into the momentum space regions originally occupied by projectile or target. ${ }^{5}$ More recently, a phase space Pauli blocker has been developed for the VUU theory. 6 We will discuss its importance below.

The Yariv-Fraenkel cascade and the Cugnon cascade differ furthermore in that

(a) the particles in the Yariv-Fraenkel simulation sit in a potential well of constant depth $V_{0}$; hence, the nuclear binding is included. This is similar to the recent cascade approach of Kitazoe et al. ${ }^{13}$ Binding has also been included into the Cugnon cascade by freezing ${ }^{14}$ the Fermi motion of each nucleon until it collides with another particle.

(b) in the original Yariv-Fraenkel approach the projectile nucleons are cascading through the target medium. In the more recent, updated version, this scheme has been improved by including the so-called cascade-cascade interactions: for a given cascade particle (a particle which has undergone at least one collision), the other cascade particles are acting as a medium superimposed to the original target medium.

In spite of the differences between the two codes, they make very similar predictions for observables like the proton and pion cross sections. In fact, they have both been shown to substantially overpredict the observed pion multiplicities in $\mathrm{Ar}+\mathrm{KCl}$ collisions. ${ }^{2,15,6}$ These deviations have recently been connected to effects of the compression energy not present in the cascade model. ${ }^{15,6}$ Of course, one has to examine carefully whether problems other than the missing compression energy are present in the cascade code. There is, for instance, the influence of the Pauli principle, which is handled in the present cascade codes in a very simple manner. We will come to this point again below.

If the two approaches are so similar, then what is the origin of the differences between the flow angles calculated with Cugnon's code ${ }^{1}$ or Kitazoe's $\operatorname{code}^{13}$ and the flow angles calculated with the Yariv-Fraenkel code ${ }^{3}$ as well as with the bound version of Cugnon's cascade code ${ }^{14,6}$

\section{FLOW ANALYSIS}

To study the origin of these discrepancies, we have performed 4000 intranuclear cascade simulations of the reaction $\mathrm{Nb}(400 \mathrm{MeV} /$ nucleon $)+\mathrm{Nb}$ with impact parameters up to $4 \mathrm{fm}$. We have employed Cugnon's code in the unbound $^{2}$ and bound ${ }^{14}$ version, where the deltas have finite lifetimes and pions can interact with the nucleons (unlike the Kitazoe code, ${ }^{13}$ which includes only instantaneous pion production and neglects pion absorption). The individual events were analyzed using the coalescence invariant kinetic energy flow tensor: ${ }^{14}$

$$
K_{i j}=\sum_{v} p_{i}(v) p_{j}(v) / 2 m(v)
$$

The sum is over the charged particles observed in each individual event and the indices $(i, j)$ represent the Cartesian components $(x, y, z)$. The tensor is diagonalized and the flow angle $\theta_{F}$ obtained via a principal axis transformation. Then flow angle distributions, which incorporate the proper Jacobian, ${ }^{16}$ are made. There are two issues where particular care must be taken in interpreting these flow angle distributions and comparing them to the experimental data. Firstly, there is the stability or binding of the nuclei. Secondly, the theoretical predictions have to be subjected to the acceptance windows and efficiency cuts imposed by the plastic ball ${ }^{3}$ (experimental filter).

\section{NUCLEAR INSTABILITY}

Concerning the issue of nuclear instability, Cugnon et al. had noted in their original paper ${ }^{2}$ that the nuclei do expand as a result of the Fermi momenta of the nucleons since a mean field is absent in the Cugnon cascade. This is illustrated quite dramatically in Fig. 1(a) for $\mathrm{Nb}$ $\left(E_{\text {lab }}=0\right)+\mathrm{Nb}$, i.e., the system (at rest) for which Cugnon and L'Hôte ${ }^{1}$ report collective flow at $400 \mathrm{MeV} /$ nucleon. Notice that the two nuclei, which are at rest and do not collide, become completely obliterated over the course of a typical collision time $t=40 \mathrm{fm} / c$. There is a rapid expansion of the original nuclei, which are just supposed to sit there. The fact that there is a problem for massive nuclei has been pointed out several years ago, ${ }^{14}$ but not much attention was paid to a solution, because predominantly light systems at higher energies were studied then, where the expansion does not play such a dramatic role because of the shorter collision time.

How about the other models that have exhibited collective flow? Is it possible that some of the flow that is observed there is also due to instability and the Fermi momenta? Nuclear fluid dynamics, the classical equations of motion approach, the Vlasov-Uehling-Uhlenbeck theory, and the time dependent Dirac-Hartree theory all have either forces or a mean field which serves to bind the nu- 


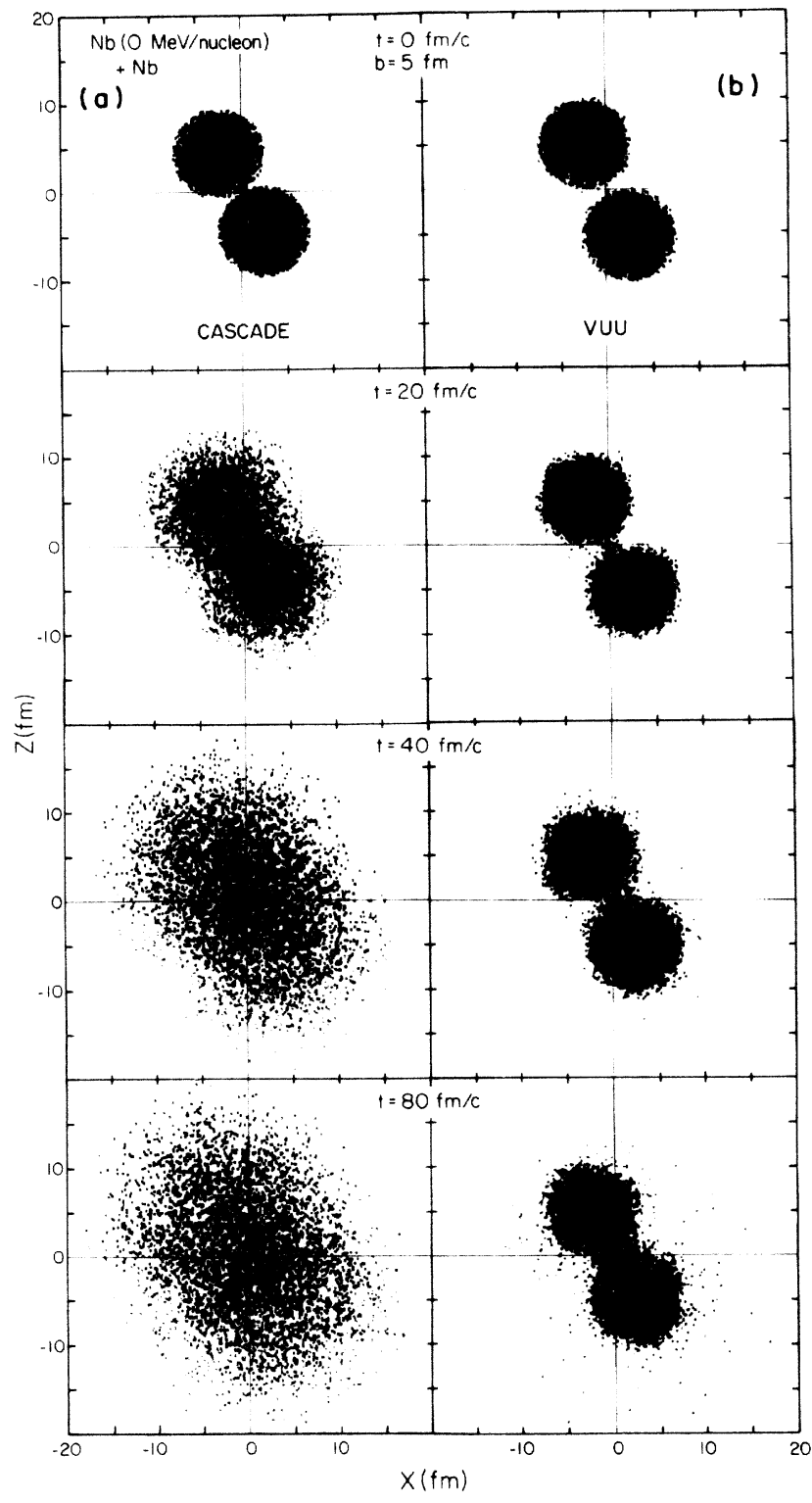

FIG. 1. Evolution of the distribution function in configuration space for $\mathrm{Nb}+\mathrm{Nb}$ at $b=5 \mathrm{fm}$ at rest, i.e., at $E_{\text {lab }}=0$ (zero) $\mathrm{MeV} /$ nucleon, as a function of time: (a) left-instability of nuclei in the unbound cascade model as used by Cugnon and L'Hôte; (b) right-the same calculation done with the VlasovUehling-Uhlenbeck approach (Refs. 6, 8, and 18).

cleons. Thus the predictions of all these theories are not invalidated due to nuclear instability. This is shown for the Vlasov-Uehling-Uhlenbeck theory in Fig. 1(b). We have let ensembles of two $\mathrm{Nb}$ nuclei at $E_{\text {lab }}=0$ (zero) $\mathrm{MeV} /$ nucleon evolve for $80 \mathrm{fm} / c$, just as was done for the Cugnon unbound cascade in Fig. 1(a). Note that only a few particles are evaporated during this time.

It is possible to bind the nucleons within the context of the Cugnon code by letting each nucleon move only with the beam velocity until it interacts with another nucleon, at which point it "remembers" its Fermi motion (if not hit at all, the Fermi motion is given to the nucleon at the end of the reaction). ${ }^{14}$ Of course, this bound Cugnon cascade does not exhibit the nuclear instability or deleterious expansion that the (unbound) original code does.

The procedure for introducing the binding is not unique and has been introduced differently in Ref. 14 and in the recent Ref. 21. The general strategy in the Cugnon cascade is first to determine the time $t(a, b)$ after which the next nucleon-nucleon collision will occur. (This can be predicted once the instantaneous positions and momenta of the particles are known.) Then the nucleons are propagated for the time span $t(a, b)$. The next step is to compare the distance $d(a, b)$ with the cross section $\sigma(s)$ :

$$
\pi d^{2}(a, b)<\text { or }>\sigma(\sqrt{s}),
$$

where $\sqrt{s}$ is the c.m. energy. In the unbound version all the three steps are made with the kinematics indicated by the Fermi motion. In Ref. 14, the first step, i.e., the determination of the $t(a, b)$ is made with the unbound kinematics whereas the second step is performed with the bound kinematics. The determination of $d(a, b)$ is done with the same kinematics. Finally the evaluation of $\sqrt{s}$ is made with the unbound Fermi kinematics. In Ref. 21 all the manipulations are done with the bound kinematics, except for the calculation of $\sqrt{s}$. It is shown in Ref. 21 that the two ways of introducing binding are largely equivalent, except for very small $b<1 \mathrm{fm}$. In this case, the method of Ref. 14 gives no flow. In contrast the method of Ref. 21 gives larger flow angles (see below). This sensitivity to the way of "binding" for very small impact parameters (despite their little weight for ordinary observables) has not been recognized until now. Flow angle distributions for $\mathrm{Nb}(400 \mathrm{MeV} /$ nucleon $)+\mathrm{Nb}$ have been calculated with these two different bound ${ }^{14,6,21}$ and unbound $^{1,2}$ versions of Cugnon's code. ${ }^{2}$ It has been found that the original-unbound-version of the cascade exhibits sidewards flow. ${ }^{1}$ This sidewards flow is reduced ${ }^{6}$ when the binding of the spectator nucleons is taken into account. It should be noted that the peak position of the flow angular distribution does not depend strongly on the impact parameter, neither for the bound ${ }^{14}$ nor the unbound cascade, in sharp contrast to what is predicted by models which incorporate the nuclear compressional energy explicitly. ${ }^{4,6-8}$ In fact, the flow angle distributions obtained with the bound cascade ${ }^{14}$ are always peaked at angles of about twelve degrees or less. This is the obvious forward peaking which has been observed by other authors $3,4,6,14,16-19$ using the revised bound Cugnon code or the Yariv-Fraenkel cascade. Note, however, that the bound cascade is theoretically not satisfactory either, since in real nuclei, nucleons can travel in all directions. A self-consistent treatment of the nuclear binding and compression potential is required for a more realistic description of the reaction dynamics, for instance in the manner of the Vlasov-Uehling-Uhlenbeck approach. ${ }^{6,8,17-19}$

\section{FLOW OF SPECTATORS AND PARTICIPANTS}

Can we understand from the above the origin of the larger sidewards flow in the unbound cascade? The nu- 


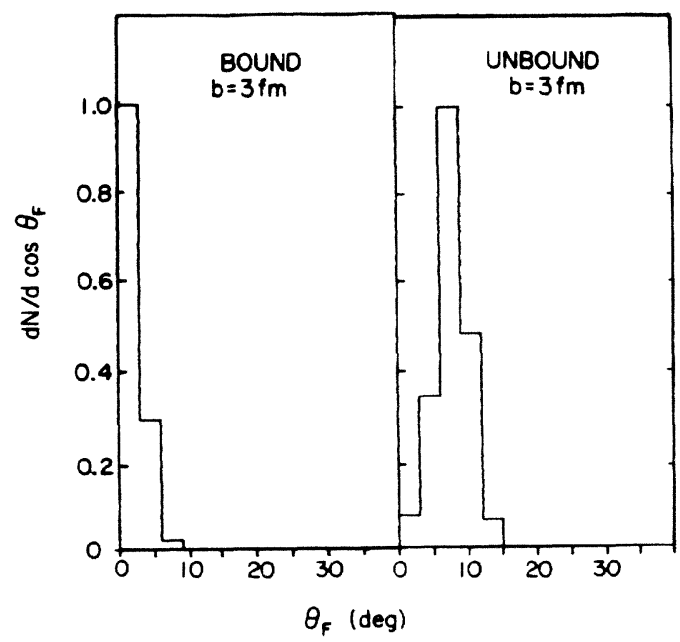

FIG. 2. Spectator flow angle distributions-include only those particles which have undergone zero collisions, the spectator nucleons, for $\mathrm{Nb}(400 \mathrm{MeV} /$ nucleon $)+\mathrm{Nb}$ at $b=3 \mathrm{fm}$ as resulting from the bound and the unbound cascade code, respectively. The binding method of Ref. 14 has been applied.

cleons in each unstable nucleus, which move towards the other nucleus due to their Fermi motion, have a large probability of colliding. Hence they tend to form a more or less equilibrated hot participant system (fireball). On the other hand, those nucleons which expand freely away from the beam axis do generate finite flow angles because the projectile expands into the upper hemisphere and the target into the lower hemisphere. This creates an artificial nondynamical flow effect in the unbound cascade.

To study this point further, we have performed the flow analysis for only those nucleons which have not undergone any collisions (the spectators). The results are shown in Fig. 2 for $\mathrm{Nb}(400 \mathrm{MeV} /$ nucleon $)+\mathrm{Nb}$ using the bound ${ }^{14}$ and unbound cascades at $b=3 \mathrm{fm}$. Notice that the spectator nucleons flow in the unbound cascade, whereas the revised code ${ }^{14}$ exhibits forward peaking. Figure 3(a) shows the results for the participant nucleons, i.e., those particles which have undergone at least one collision (the spectator nucleons shown in Fig. 2 are removed from the analysis). Here we have also started to employ the efficiency and multiplicity filter SIMDAT discussed below. The flow angles of the participants are also smaller for the bound cascade ${ }^{14}$ than for the unbound one. But in both cases the flow is substantial. This result therefore clearly establishes that the two-body collisions, which dominate the cascade dynamics, can on their own produce finite flow effects.

Figures 3(a) and (b) show that the participants do yield a finite sidewards flow-the flow effect is not solely due to the treatment of the spectators in the unbound cascade. However, even the substantial isolated participant flow is not sufficient to explain the large angle sidewards maxima observed experimentally. ${ }^{3}$ What is causing this flow of the participants in the cascade calculation? It is the finite amount of pressure buildup in the interaction zone, which pushes on layers of matter through subsequent collisions.
The additional strong repulsive interactions present in the other theories are, however, missing in the cascade approach. That there is a finite pressure buildup in the cascade can be seen when the diagonal elements of the stress tensor in central collisions are evaluated near the center of mass. $^{21}$

It is interesting to note that the flow of the participants can be enhanced by unbinding the nuclei [see Fig. 3(a)]. A possible explanation of this fact could be the following: because of their Fermi motion, some additional nucleons move into the interaction zone. This is actually reflected in an increased number of their binary collisions. The interaction zone thus would be less transparent-for these nucleons and the still undisturbed remnants, which have not yet entered the interaction region. Additional pressure buildup would occur. It is amusing to note that for $b=0 \mathrm{fm}$ the situation is somewhat different: In the case $^{14}$ more nucleons are moving away from the interaction region, thus decreasing the chance for collisions and making the participant region more transparent. Therefore, the maximum flow is obtained in the unbound cascade for impact parameters around $3 \mathrm{fm}$, while for exactly central collisions, $b=0 \mathrm{fm}$, the flow angular distribution is peaked at zero degrees, even for the unstable cascade.

However the increasing chance for escaping depends on the way the binding is done. That is the reason why the participant zone is less transparent in Ref. 21 compared to the results obtained with the method of Ref. 14 .

Figure 3(c) shows the impact parameter dependence of the flow, both in the bound (here with the method of Ref. 21) and the unbound versions. As we have already indicated above, the flow is not changing very much in this range of impact parameters. For more central impact parameters, the flow angle will increase very much for the method of Ref. 21 and will go to zero for the method of Ref. 14. This diverging behavior is not very significant for a comparison to experimental data, however, since these very central collisions are not very frequent and since these events have a very spherical shape.

\section{CAN THE CASCADE APPROACH THE VISCOUS FLUID LIMIT}

Can the intranuclear cascade model predict-at least in principle, say by going to very massive systems-the same large flow angles as the data or the hydrodynamic model? In the limit of a short mean free path, the cascade model should approach the hydrodynamic limit and the results of cascade and hydrodynamics should be similar. But even for $U+U$ collisions, the standard Cugnon cascade produces near isotropy and does not approach the fluid flow limit as was shown by Gyulassy, Frankel, and Stöcker. $^{14}$ Calculations with $A_{P}=A_{T}=500,1000$, and even 2000 confirm this finding. ${ }^{14}$

Can fluid behavior be forced into the cascade by using larger effective NN cross sections to induce fluid behavior? This has also been tried previously. ${ }^{14}$ But, although the flow angle increases somewhat, the flow is still far weaker than the hydrodynamic result. ${ }^{14}$ To understand this puzzling finding we must remember that the hydrodynamic calculations have-in addition to the as- 
(a)

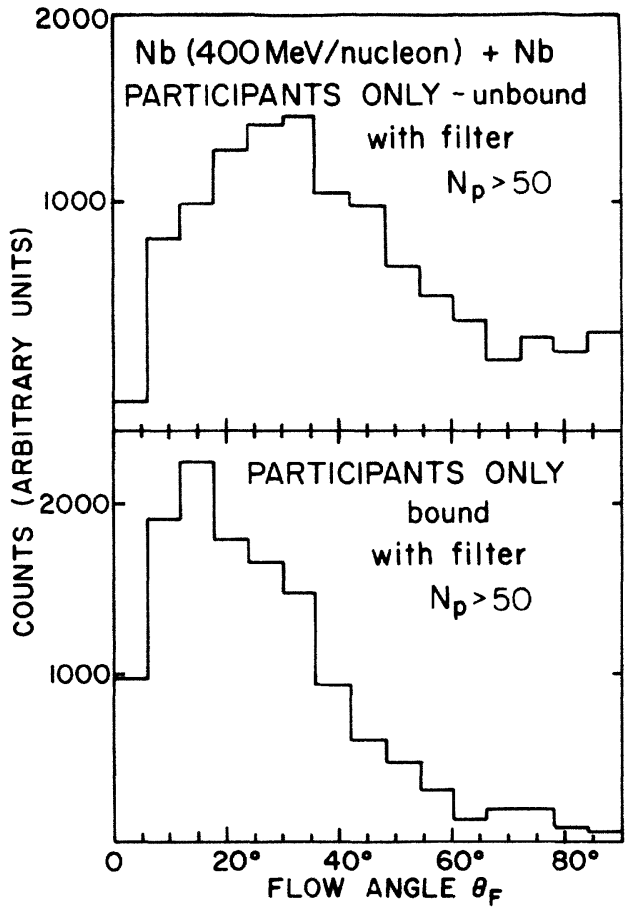

(b)

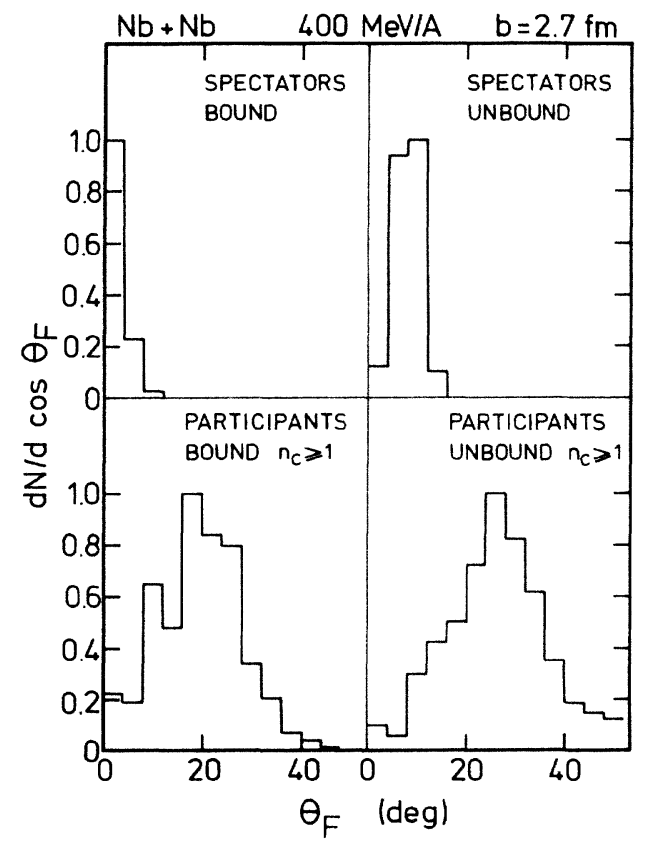

(c)

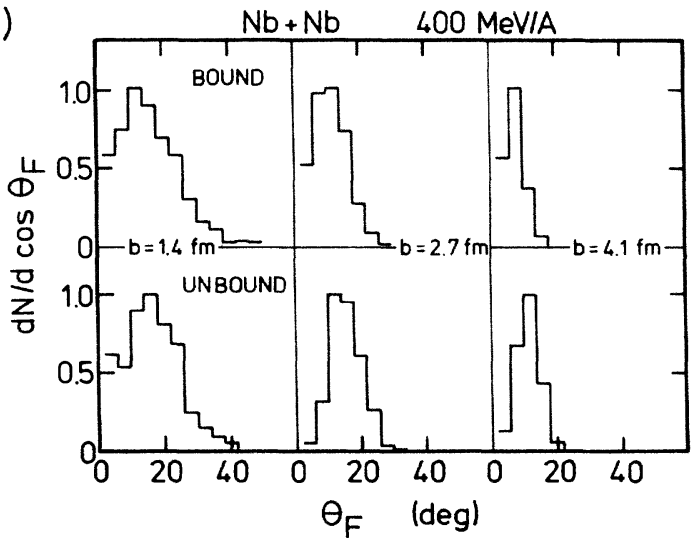

sumption about a short mean free path-also incorporated a repulsive short-range nuclear interaction via a compressional potential in the nuclear equation of state. Hence, $\lambda / R \ll 1$ is not sufficient to simulate the observed fluid behavior.

It has been shown ${ }^{14}$ though, that the flow is sensitive to the scattering style-recall that the standard scattering style corresponds to a stochastic classical force with a random sign-"inward" scattering occurs with the same probability as "outward" scattering. Therefore, the momentum transfer and the relative coordinate between two nucleons are assumed to be completely uncorrelated at the scattering time. Classically, on the other hand, any potential leads to definite correlations between $r$ and $p$. Some of these correlations have been implemented into the standard Cugnon cascade. ${ }^{14}$ It has been shown that only the nonrandom scattering (repulsive in-plane scattering) with increased scattering cross section leads to collective flow similar to nonviscous hydrodynamics, as was later observed in the classical equations of motion approach which incorporates an excluded volume approximation. ${ }^{7}$ As the effective cross section is increased, the flow becomes more pronounced. ${ }^{14}$ For five times normal scattering cross section, the flow is even more pronounced than in nonviscous fluid dynamics with a soft equation of state. Thus, the flow obtained with nonviscous one-fluid hydrodynamics represents only one possible class of flow patterns.

Gyulassy, Fraenkel, and Stöcker have conjectured ${ }^{14}$ that the variations of the effective scattering cross section and scattering style correspond to substantial variations in the transport properties and the equation of state in terms of viscous fluid dynamics. Therefore, it seems to be of utmost importance to include the short-range nuclear repulsion into any microscopic theory of medium and high energy nuclear phenomena, e.g., via the repulsive nuclear compression potential fields which are neglected in the intranuclear cascade model.

\section{COMPARISON TO EXPERIMENTAL DATA- INFLUENCE OF INSTRUMENT EFFICIENCY AND MULTIPLICITY CUTS}

We must remember to examine the effect of the acceptance windows and efficiency cuts of the plastic ball in or-

FIG. 3(a). Participant flow angle distributions-include only those particles which have undergone at least one collision, the participant nucleons, for $\mathrm{Nb}(400 \mathrm{MeV} /$ nucleon $)+\mathrm{Nb}$. In contrast to the previous picture we have applied here the experimentalists's (Ref. 3) SIMDAT efficiency and multiplicity procedure to the theoretical "data" as resulting from the bound and the unbound cascade code, respectively. The binding method of Ref. 14 has been applied. (b) Spectator (upper part) and participant (lower part) flow angle distributions, for $\mathrm{Nb}(400$ $\mathrm{MeV} /$ nucleon $)+\mathrm{Nb}$ at $b=2.7 \mathrm{fm}$ as resulting for the bound and the unbound cascade code, respectively. The method of Ref. 21 has been used. (c) Total (participants-plus spectators) flow angle distribution for intermediate impact parameters in the $\mathrm{Nb}(400 \mathrm{MeV} /$ nucleon $)+\mathrm{Nb}$ system. The upper part refers to the bound version of the cascade and the lower part to the unbound version of Ref. 21 . 
der to compare the theoretical and experimental flow distributions in the same multiplicity bins. We have used the original SIMDAT data simulation routine developed by the GSI/LBL plastic ball group to simulate the response of their spectrometer system to Monte Carlo events. In the SIMDAT routine the cascade nucleons are randomly assigned isospin, target nucleons which have not collided are omitted, and the residual charged particles are subjected to the acceptance and efficiency of the plastic ball, most notably a lower and upper threshold for particle identification in the kinetic energy of about 20 and 200 $\mathrm{MeV} /$ nucleon, respectively. The composite particles are not treated explicitly and this could introduce some uncertainty in the final result, since the experimental cuts depend to some extent on the nature of the particle. Then the events at finite impact parameters are binned according to the multiplicity of charges that the plastic ball would see. Notice that there is a strong dependence of the impact parameter distribution on the selected multiplicity bin as is illustrated in Fig. 4.

The invariant cross sections $d^{2} \sigma / d Y d\left(p_{x} / m\right)$ in the scattering plane, which are determined from the finite multiplicity cascade events on an event by event basis, are shown in Fig. 5 for the multiplicity bin $40<M<50$ for the bound and unbound cascade, respectively. $Y$ is the rapidity and $p_{x}$ is the transverse momentum component in the reaction plane. Observe the strong depletion of the cross section near the target rapidity: This is due to the absorption of low energy particles in the wall of the scattering chamber in the plastic ball, which is here simulated by the SIMDAT routine. Note that the contour plot for the bound cascade is more symmetric about the beam axis than the unbound cascade contour plot, again reflecting the reduced flow in the bound cascade. ${ }^{14}$

We now come to the most important result of this paper: The flow angle distributions for the bound and unbound versions of the code by Cugnon et al., subjected to the plastic ball filter and binned according to multiplicity, are shown in Fig. 6. Also shown are the experimental

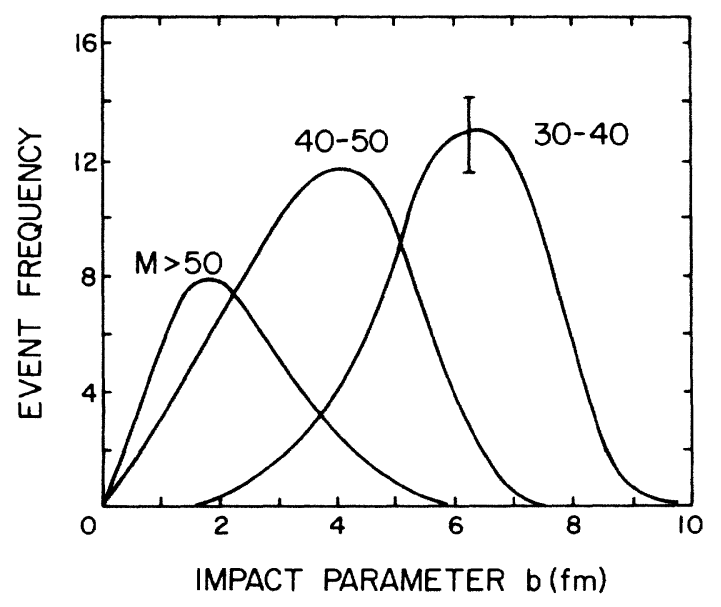

FIG. 4. Dependence of the impact parameter distribution on the multiplicity bin chosen, if the bound cascade results are subjected to the plastic ball filter SIMDAT.

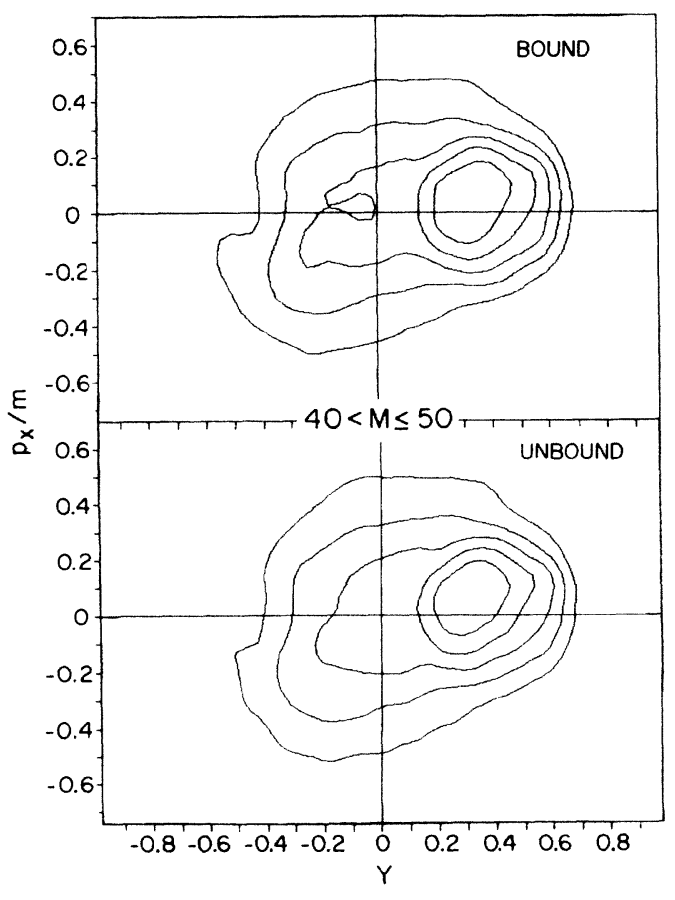

FIG. 5. Invariant cross section $d^{2} \sigma / d Y d\left(p_{x} / m\right)$ for $\mathrm{Nb}(400$ $\mathrm{MeV} /$ nucleon) $+\mathrm{Nb}$ in the reaction plane for $40<M<50$ for the bound and the unbound cascade, respectively.

data $^{3}$ for the two highest multiplicity bins. Note that neither the bound nor unbound cascade match the $\mathrm{Nb}(400$ $\mathrm{MeV} /$ nucleon) $+\mathrm{Nb}$ data. We can compare the results of Refs. 1 and 6, which show the flow distributions without filter, with our Fig. 6, which includes the effects of the filter and the multiplicity selection (rather than impact

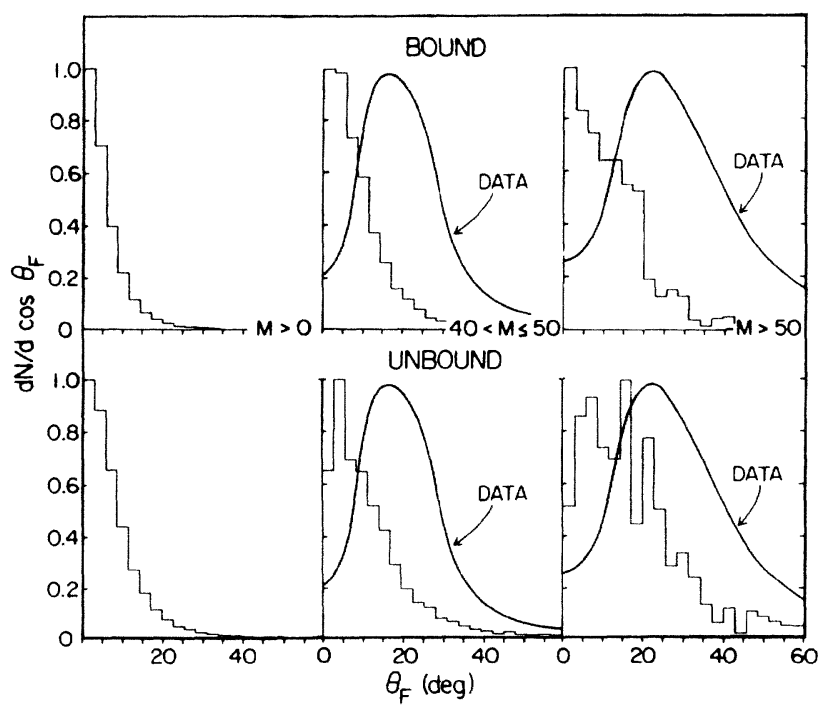

FIG. 6. Flow angle distribution for $\mathrm{Nb}(400$ $\mathrm{MeV} /$ nucleon) $+\mathrm{Nb}$ binned according to the multiplicity of charges seen by the plastic ball for bound and unbound cascade, respectively. Also shown are the experimental data for the two highest multiplicity bins. 
parameters, which cannot be accessed experimentally). The comparison shows that the filter and the multiplicity selection used here tend to cause the flow angle distribution to broaden, but they do not increase the peak angle substantially. Notice, however, that the filter includes the rejection of the target spectators. This rejection tends to increase the peak angle.

Several remarks concerning the filter are in order: apparently there is an effect of the filter on the resulting angular distributions; also the multiplicity of charged fragments is not unambiguously obtained from the cascade model (the multiplicity of charges is more easily accessible), unless one takes account of both the target spectators (as we have done explicitly here) and of cluster formation (which we have not done, although one can use a sixdimensional coalescence of the final state ${ }^{20}$ ). Therefore it is not immediately obvious which multiplicity binning should be chosen to compare the data with the theory.

However, we can rectify this problem by rebinning the data according to the multiplicity of charges (rather than according to the multiplicity of charged fragments, as was done in Ref. 3); then there is no significant change of the flow distributions in the same multiplicity bin. Notice, however, that there is still some ambiguity in the calculation of the charge multiplicity produced by the cascade. For instance, the energy that a proton needs to have to be identified in the detector depends to some degree on whether it is free or bound in a composite. Obviously, in the data there is a substantial effect of the multiplicity bin to its flow angle distribution-going to larger multiplicities results in increasing flow angles.

To illustrate this point further we have applied a very high multiplicity cut $(M>66)$ to the bound cascade ${ }^{21}$ results, using the simple filter described in Ref. 1. Then a maximum in the flow distribution is observed, which is located approximately at the peak position observed in the experimental data for multiplicities around 50. If the multiplicity cut $M$ on the cascade results is lowered, the peak moves to even smaller angles, as shown above. It would be desirable to have information about the detailed experimental cross sections of the emitted fragments in the different multiplicity bins and for the cluster formation and spectra to be self-consistently calculated in the theory.

This could allow for a quantitative determination of the difference between the flow produced by cascade and the experimental one. The difficulty in comparing cascade results to experimental data comes from two origins. The first concerns the composites: as the experimental flow includes bound neutrons, a procedure to calculate composite production must be added to the cascade. The final flow can then depend to some extent on this procedure. The second one, as was already mentioned, concerns the experimental cuts and multiplicity calculation.

These considerations could explain the difference between the flow distributions obtained in Ref. 1 and the unbound cascade ones presented here in Fig. 6. In Ref. 1, the highest multiplicity bin $(M>62)$ is different from ours $(M>50)$, but the two multiplicities are calculated differently. In addition, the experimental filter used in Ref. 1 rejects target nucleons on the basis of energy transfer suffered during the collision process, while the SIMDAT filter used here rejects all uncollided target nucleons plus those which would have too little energy to reach the plastic detectors as well as those nucleons which have too high an energy and would punch through the detector. Hence, a target nucleon that received a small energy transfer, but whose Fermi momentum is large, will be rejected by the filter of Ref. 1 and accepted by the experimentalists's filter used here.

Nevertheless, we point out that no reasonable variation on the experimental filter applied to the cascade can produce flow angles as large as the experimentally observed ones.

We make a final remark that there is a need to understand how the plastic ball filter operates on the physics. Indeed in Ref. 1, it is shown that the two main operations of the filter, namely the removal of the neutrons and the elimination of the target spectators, are expected to increase (slightly, but increase anyhow) the flow angle. The use of SIMDAT seems to indicate that the effects of the details of the filter are far from being understood. For instance, it may very well be that a nonisotropic distribution of the clusters or the "double hits" have an effect which is more peculiar than expected at first thought. This deserves further investigation.

\section{INFLUENCE OF PAULI BLOCKING AND REARRANGEMENT ON FLOW CALCULATIONS}

It is remarkable how similar the results from the bound cascade obtained here are to those which the experimentalists obtained ${ }^{3}$ using the Yariv-Fraenkel code. This underlines the fact that the presence of a constant depth potential does not lead necessarily to dynamical flow. The claim that including a potential well could give a contribution to the flow in the calculation of Kitazoe et al. ${ }^{13}$ needs further investigation: There are differences between the input of the codes of Cugnon et al. and Kitazoe et al., as discussed in Ref. 13:

(a) without a collision, the nuclei are quite stable in the Kitazoe approach and there is only negligible nucleon emission, in spite of the presence of Fermi motion-this is, however, similar to the Yariv-Fraenkel cascade, which does not exhibit large flow angles;

(b) projectile and target residues and their c.m. velocities are well defined and change gradually as a function of time-this is again similar to Ref. 5;

(c) soft nucleon-nucleon collisions are not prohibited, which-according to the authors of Ref. 13-increases the cascade density;

(d) there is a substantial number of reflections of nucleons at the nuclear surface, which-according to the authors of Ref. 13-increase the cascade density and are responsible for the stability of the initial nuclei.

We have pointed out above that the "observable" sidewards peak, which is obtained by applying the plastic ball filter to the raw theoretical "data," disappears by applying the binding in Cugnon's code. Therefore the peak should also not appear in Kitazoe's approach. However, Kitazoe et al. obtain sidewards peaking ${ }^{13}$ which reportedly is con- 
sistent with the data, while the peaks are positioned at too small angles even in the unbound Cugnon code (Fig. 6). It could be possible that Kitazoe et al. do obtain sidewards flow because of an instability of their nuclei which is caused by the drastic readjustment of the nuclear radius during a collision event.

This could result from their stringent requirement that the nuclear density is not allowed to be depleted, as is the case in the Yariv-Fraenkel cascade, but is bound to stay constant. The radius of their residual nucleus therefore shrinks rapidly as collisions between nucleons occur, thus shaking off a substantial amount of uncollided nucleons from the residual nuclei. A quantitative analysis of this effect is not possible at this time, because the computer code $^{13}$ has not been accessible to the authors.

We would like to point out that the rearrangement of the nuclear density distribution can have a strong influence (factors of 2-3) already on inclusive proton distributions as observed by Yariv and Fraenkel. ${ }^{5}$

The rearrangement of the nuclear density distribution is of course intimately related to the effects of the Pauli principle on the elementary scattering processes. We have checked the influence of the Pauli principle on the flow angular distributions by employing the phase space Pauli blocker developed for the VUU approach. ${ }^{6,20,18,19}$ It differs from the simple minimum relative energy Pauli blocker employed by Cugnon, and the Pauli blocking of the projectile and target region used in Yariv and Fraenkel's as well as in Kitazoe's code: the phase space density $f$ is determined in a six-dimensional sphere centered around the center of mass for each individual twobody scattering event. The Pauli blocking probability for such an attempted collision is then proportional to $\left(1-f_{1}\right)\left(1-f_{2}\right) .^{6,18-20}$

Then, collisions can be prohibited even if the relative velocity of the nucleons is large and the final state of the scattered particles is located in the midrapidity region, provided only the midrapidity region is partially filled. This is also responsible for the rather weak flow effects seen in the $\mathrm{Ca}+\mathrm{Ca}$ system, ${ }^{3}$ where the small size of the system barely allows for equilibration and compression, but does not result yet in a drastic collective sidewards flow ${ }^{3,6,8}$ (see, however, the work of Danielewicz and Odiniec, Ref. 3, who report isolation of collective flow effects even in these lighter systems). We find ${ }^{17-19}$ that the peak flow angle for $\mathrm{Nb}+\mathrm{Nb}$ can be reduced by as much as a factor of 2 (depending on the incident energy and impact parameter) when this more realistic Pauli blocking procedure is applied. ${ }^{19}$ That means that our present cascade results, as well as the cascade results of other groups, must be taken with caution-the present calculations probably still overestimate the flow effects achievable in the cascade approach.

\section{CONCLUSIONS}

In summary, we have shown that Cugnon's intranuclear cascade model lacks dynamical flow. Flow effects had been overestimated previously due to spurious expansion of the spectators. When binding is taken care of, the cascade model does exhibit some flow. The latter comes from the pressure which is inevitably built up inside the system through the binary collisions. However, when experimental efficiency and multiplicity cuts are imposed on the cascade "data" the flow is considerably masked. In spite of the uncertainties associated with the filtering of the theoretical events and in particular of the unsolved problem of the composite formation, the resulting flow in the cascade is definitely too small when compared to experiment. Therefore, we conclude that a satisfactory explanation of the observations needs a physics not contained in the cascade, namely compression energy and a proper account of the quantum statistics. This is naturally included in the Vlasov-Uehling-Uhlenbeck approach. The latter has been successfully applied to study the influence of the nuclear compression potential and the Pauli principle. $6,8,17-19$

From a comparison to the experimental data, $3,15,16$ more and more convincing arguments are accumulated in favor of a rather stiff equation of state. $6,8,17-19$

\section{ACKNOWLEDGMENTS}

We express our sincere gratitude to the members of the GSI/LBL plastic ball group, in particular Dr. HansGeorg Ritter, for help in analyzing the effect of the acceptance and efficiencies of the plastic ball detector system on the theoretical data. We also acknowledge the help of Randy Rencsok in converting the programs for use on the FPS-164 attached processor at the MSU-NSCL's computing center and in demonstrating the stability of the VUU approach.
"Permanent address: Institut für Theoretische Physik, Johann Wolfgang Goethe Universität, D-6000 Frankfurt am Main, Federal Republic of Germany.

†Permanent address: Department of Physics, University of Lund, S-22362 Lund, Sweden.

†Permanent address: Physique Nucleaire Theoretique, Universite de Liège, B-4000 Sart-Tilman, Liège, Belgium.

${ }^{1}$ J. Cugnon and D. L'Hôte, Phys. Lett. 149B, 35 (1984); and (unpublished).

2J. Cugnon, Phys. Rev. C 22, 1885 (1980); J. Cugnon, T. Mizutani, and J. Vandermeulen, Lett. Nuovo Cimento 28, 55
(1980); Nucl. Phys. A352, 505 (1981); J. Cugnon, D. Kinet, and J. Vandermeulen, ibid. A379, 553 (1982).

${ }^{3}$ H. A. Gustafsson, H. H. Gutbrod, B. Kolb, H. Löhner, B. Ludewigt, A. M. Poskanzer, T. Renner, H. Riedesel, H. G. Ritter, A. Warwick, F. Weik, and H. Wieman, Phys. Rev. Lett. 52, 1590 (1984); H. G. Ritter et al., Proceedings of the II International Conference on Nucleus-Nucleus Collision, Visby, Sweden, 1985, Nucl. Phys. A, in press. Similar conclusions have been obtained for asymmetric collision systems at the LBL streamer chamber: R. Renfordt, D. Schall, R. Bock, R. Brockmann, J. Harris, A. Sandoval, R. Stock, H. Stroebele, 
D. Bangert, W. Rauch, G. Odiniec, H. G. Pugh, and L. S. Schroeder, Phys. Rev. Lett. 53, 763 (1984). A novel transverse momentum analysis has also revealed evidence for much stronger flow than predicted by the Cugnon cascade; see, $\mathbf{P}$. Danielewicz and G. Odiniec, Phys. Lett. 157B, 146 (1985) and Ref. 8.

${ }^{4}$ H. Stöcker, J. A. Maruhn, and W. Greiner, Phys. Rev. Lett. 44, 725 (1980); G. Buchwald, G. Graebner, J. Theis, J. Maruhn, W. Greiner, and H. Stöcker, ibid. 52, 1594 (1984); Phys. Rev. C 28, 2349 (1983).

${ }^{5}$ Y. Yariv and Z. Fraenkel, Phys. Rev. C 20, 2227 (1979); 24, 488 (1981).

${ }^{6}$ H. Kruse, B. V. Jacak, and H. Stöcker, Phys. Rev. Lett. 54, 289 (1985).

7J. J. Molitoris, J. B. Hoffer, H. Kruse, and H. Stöcker, Phys. Rev. Lett. 53, 899 (1984); S. M. Kiselev, Phys. Lett. 154B, 247 (1985); R. Malfliet, Phys. Rev. Lett. 53, 2386 (1984).

${ }^{8}$ J. J. Molitoris and H. Stöcker, Phys. Rev. C 32, 346 (1985); J. J. Molitoris and H. Stöcker, Phys. Lett. 162B, 47 (1985).

${ }^{9}$ R. Y. Cusson, P. G. Reinhard, J. J. Molitoris, H. Stöcker, M. R. Strayer, and W. Greiner, Phys. Rev. Lett. 55, 2786 (1985).

${ }^{10}$ R. Serber, Phys. Rev. 72, 1114 (1947).

${ }^{11}$ M. L. Goldberger, Phys. Rev. 74, 1269 (1948).

${ }^{12}$ N. Metropolis, R. Bivins, M. Storm, A. Turkevitch, J. M. Miller, and G. Friedlander, Phys. Rev. 110, 185 (1958). Other cascade approaches, which have not been to our disposal and are therefore not discussed in detail here, include J. D. Stevenson, Phys. Rev. Lett. 41, 1702 (1978); E. C. Halbert, Phys. Rev. C 23, 295 (1981); V. D. Toneev and K. K. Gudima, Nucl. Phys. A400, 173 (1983).
${ }^{13}$ Y. Kitazoe, H. Furutani, H. Toki, Y. Yamamura, S. Nagamiya, and M. Sano, Phys. Rev. Lett. 53, 2000 (1984); Phys. Rev. C 29, 828 (1984).

${ }^{14}$ M. Gyulassy, K. A. Fraenkel, and H. Stöcker, Phys. Lett. 110B, 185 (1982); J. W. Harris (unpublished).

15J. W. Harris, R. Stock, R. Bock, R. Brockmann, A. Sandoval, H. Strobele, G. Odiniec, H. G. Pugh, L. S. Schroeder, R. E. Renfordt, D. Schall, D. Bangert, W. Rauch, and K. L. Wolf, Phys. Lett. 153B, 377 (1985); R. Stock et al., Phys. Rev. Lett. 49, 1236 (1982). See, H. Stöcker, W. Greiner, and W. Scheid, Z. Phys. A 286, 121 (1978) for a first prediction of the dependence of the pion yields on the nuclear compression energy.

${ }^{16}$ P. Danielewicz and M. Gyulassy, Phys. Lett. 129B, 283 (1983).

${ }^{17}$ J. J. Molitoris and H. Stöcker, Proceedings of the 7 th High Energy Heavy Ion Study, Darmstadt, Germany, 1984; Gesellschaft für Schwerionenforschung Report 85-10, 1985, p. 197-235; J. J. Molitoris, Ph.D. thesis, Michigan State University, 1985.

18J. J. Molitoris, D. Hahn, and H. Stöcker, Proceedings of the II International Conference on Nucleus-Nucleus Collision, Visby, Sweden, 1985, Nucl. Phys. A, in press; H. Stöcker and W. Greiner, Phys. Rep. (to be published).

${ }^{19}$ J. J. Molitoris and H. Stöcker (unpublished).

${ }^{20}$ H. Kruse, B. V. Jacak, J. J. Molitoris, H. Stöcker, and G. D. Westfall, Phys. Rev. C 31, 1770 (1985).

$21 \mathrm{~J}$. Cugnon and D. L'Hôte, Proceedings of the II International Conference on Nucleus-Nucleus Collisions, Visby, Sweden, 1985, Nucl. Phys. A (to be published); submitted to Nucl. Phys. A. 